\title{
DNA-based Animal Models of Human Disease: from Genotype to Phenotype
}

L.B. Schook ${ }^{1,2}$, K. Kuzmuk', S. Adam ${ }^{3}$, L. Rund ${ }^{1}$, K. Chen ${ }^{1}$, M. Rogatcheva ${ }^{1}$, M. Mazur ${ }^{1}$, C. Pollock ${ }^{1}$, C. Counter ${ }^{3}$

1 Laboratory of Comparative Genomics, Department of Animal Sciences, University of Illinois, Urbana, USA

2 Regenerative Biology and Tissue Engineering Theme, Institute for Genomic Biology, University of Illinois, Urbana, USA

3 Department of Pharmacology and Cancer Biology, Duke Medical Center, Durham, NC, USA

Key words: pigs, animal models, splice variants, SNPs, tumorigenesis, atherosclerosis, AT

\begin{abstract}
Biomedical research utilizes animal models to elucidate human disease processes at the cellular and molecular level and for the development of new therapies. Traditionally, mammalian models have been limited to the mouse, primarily because of well characterized genetic lines and the ability to manipulate the genome to directly test hypotheses regarding causal mutations and disease phenotypes. The emerging availability of genome sequences of other mammals (bovine, canine, equine, feline, and porcine) now permits utilization of the mammal in which the phenotype best approximates the human condition. Equally important is the use of somatic cell nuclear cloning (SCNT) coupled with targeted germline manipulation to create animals to resolve the molecular mechanisms of the disease state. Our efforts have focused on the pig, which has emerged as an important biomedical mammalian model due to its closer physiology to humans. The utility of porcine genetically-defined tumour, cardiovascular and neurological disease models is described.
\end{abstract}

\section{INTRODUCTION}

Public health programmes and advances in clinical medicine have increased the human lifespan from 47.3 years in 1900 to 76.9 years in 2000 [1]. Technological and medical advances have also improved the quality of life and provided prevention strategies and treatments. However, the incidence of chronic diseases, due to complex genetic and environmental interactions, has also dramatically increased in the past 
century. In fact, the majority of the top ten leading causes of death in the United States in 2000 (heart disease, cancer, stroke, chronic lower respiratory disease, diabetes, Alzheimer's disease, and renal disease) represent diseases shown to result from such interactions [2]. Resolving complex human diseases (that reflect lifestyle influences and genetic predisposition) is difficult (e.g., expenses associated with human clinical experiments and ethical issues) and thus appropriate biomedical models must be developed and validated. In the past, researchers have used two approaches to study human diseases. One strategy fully characterizes a human clinical disease and uses the most appropriate animal model based on criteria such as anatomical and/or physiological characteristics. The recent advent of techniques in genomics, transgenesis and cloning provide a new opportunity to create domesticated animals (pigs, cows, chickens, dogs) as model organisms.

The incidence of chronic diseases due to complex genetic and environmental interactions has dramatically increased [3]. Atherosclerosis, the primary cause of coronary artery disease and stroke, is a disorder with multiple genetic and environmental contributions. Successful discovery of these genetic factors requires using complementary approaches with appropriate animal models, large-scale human genetic studies, and functional experiments [4]. Although many advances have been made in understanding the genetics of atherosclerosis by using rodent and transgenic mouse models, an animal model that will permit studies on phenotypic changes not evident in the mouse is necessary. The link between the APOE gene, serum cholesterol levels, and the development of atherosclerosis has been well established in humans. Extensive association studies with disease risk have been performed for alleles of $A P O E[5]$. These studies determined that the $\varepsilon 4$ allele is associated with an increased risk for cardiovascular disease. We are investigating the potential of the pig as a model for atherosclerosis by utilizing sequence information to compare human $A P O E$ to pig $A P O E$.

The transition from basic to clinical cancer research is hampered by the lack of a genetically malleable, large animal model. In particular, preclinical imaging studies as well as studies on hyperthermia, radiation, or photodynamic therapy of tumours are limited by this lack of suitable large animal models. Mice are ideal models for genetically inducing tumours but the organs and tumours are too small for the medical devices. For example, it is virtually impossible to do magnetic resonance tomography on mice due to their small tumour size and the energy of clinical accelerators. Highresolution intensity modulated treatment in rodents is further hampered by the same problems and devices used for hyperthermia treatment and cannot be scaled down to be useful for studies in rodents. While companion animals (particularly dogs and cats) with spontaneous tumours are ideal for imaging studies and the above described therapies, it is very difficult and expensive to accrue enough animals with the desired tumour types for clinical trials. Moreover, tumours in these animals are genetically undefined and in some cases too rare to be studied [6]. In vivo cancer studies requiring large tumours would therefore be greatly aided by a genetically defined large mammal model of cancer. The pig represents such an animal. Like humans, the incidence of cancer in pigs is rare. The parallels in cancer biology between humans and pigs are even conserved at the molecular level. For example, in both species, telomerase is suppressed in a number of tissues but reactivated during cancer. Tumours can also reach sizes seen in humans, which is particularly relevant for many preclinical applications. Additionally, pigs have the added advantage of being more similar to humans in a number of aspects compared to other commonly used animal models in cancer biology [7]. For example, the size, anatomy, and physiology of the swine 
reflect that of humans in many respects, and as such the pig is often the primary biomedical model for a number of diseases, surgical research, and organ transplantation [3]. The genomic sequence homology between humans and pigs is also very high [3] and the porcine PXR protein that regulates p450 cytochrome CYP3A, which metabolizes almost half of prescription drugs in humans, is more similar to humans than, for example, the murine PRX [8].

Ataxia-telangiectasia (A-T) is a genetic disorder causing cerebellar degeneration, immune deficiency, cancer predisposition, chromosomal instability and radiation sensitivity. Among the mutations responsible for A-T, $85 \%$ represent truncating mutations that result in the production of shorter, highly unstable forms of ATM (ATmutated) protein leading to a null ATM phenotype. Several ATM-deficient mice have been created, however none reflect the extent of neurological degeneration observed in humans. In an attempt to identify an alternative animal model, we have characterized the porcine ortholog of ATM ( $p A T M)$ to support a pig model for AT.

\section{Materials ANd Methods}

\section{RNA extraction, cDNA synthesis and sequencing}

Total RNA was extracted from frozen porcine tissues using the TRIzol ${ }^{\mathbb{R}}$ reagent (Invitrogen), treated with DNase I, and purified. Samples were reverse transcribed (+RT samples) with Oligo(dT)12-18 and random primers and Superscript II reverse transcriptase. To determine the porcine $A T M \mathrm{cDNA}$ sequence, $0.5 \mathrm{~kb}$ to 1.8 $\mathrm{kb}$ overlapping cDNA fragments were amplified and sequenced. The porcine genomic sequence (AY587061) was used as a reference to design primers located in exons of the ATM gene. The $5^{\prime}$ RACE analysis was performed on total RNA extracted using the FirstChoice RLM-RACE kit. Splicing of the 5' UTR was analysed by amplifying fragments from exon 1 to exon 8 with the forward 5' GATTCCACTTGGTTGTCCAGTC 3' and reverse Race ex $8 \mathrm{R}$ primers. PCR fragments were purified using a QIAquick PCR purification kit and bidirectionally sequenced with PCR primers. Alternatively, if multiple amplicons were observed, the amplified fragments were cloned into the $\mathrm{pCR}^{\circledR} 2.1-\mathrm{TOPO}^{\circledR}$ vector system and then sequenced using M13 primers. DNA sequencing was performed with the ABI Prism ${ }^{\circledR}$ BigDye ${ }^{\mathrm{TM}}$ Terminator Cycle Sequencing Kit and analysed on an ABI 3730 automated DNA sequencer.

\section{Quantitative RT-PCR of 5' UTR ATM transcript variants}

For the identification and quantification of 5'UTR variants, quantitative RT-PCR analyses were performed on cDNA samples from porcine tissues using TaqMan 5' nuclease assays. Primers and probes were designed with Primer Express software to anneal with splice variant-specific sequences at exon-exon junctions. To provide more accurate splice variant discrimination, TaqMan ${ }^{\circledR}$ Probes with a nonfluorescent quencher and a minor groove binder at the $3^{\prime}$ end were used. In order to estimate the total amount of ATM transcripts, a probe that recognized a sequence common to all splice variants was used. The TaqMan ${ }^{\circledR}$ assay was performed using a 96-well plate format with a $20 \mu 1$ reaction mix and the ABI PRISM 7900 Sequence Detection System instrument and software. The copy numbers of splice variants (SV) per reaction was determined for each tissue and the absolute copy number was normalized for the relative amount of $18 \mathrm{~s}$ rRNA and background subtracted using the (-RT) control reactions. To quantify the porcine 18s rRNA, the TaqMan reagent Starter kit was used according to the manufacture's protocol, except that the probe-primer mix was diluted 10 fold. An absolute quantitation assay was used to estimate the copy number of each SV by interpolating their quantity from a standard curve. Standard curves were developed using serial dilutions ( $10^{1}$ to $10^{7}$ copies) of each SV standard. A standard curve was constructed for each SV using threshold cycle data plotted against the log copy numbers for the standard dilution sets.

\section{Retroviral Vector Construction}

The retroviral vectors encoding a single transgene, pBabepuro-cyclinD1 $\bullet H A$, pBabehygro-CDK4 ${ }^{\text {R24C }} \cdot H A$, pBabeblasto-c-Myc ${ }^{\mathrm{T} 8 \mathrm{~A}}$, pBabeneo-p53 ${ }^{\mathrm{DD}}$, pBabepuro-flag $\bullet H-$ Ras $^{\mathrm{G} 12 \mathrm{~V}}$ and $\mathrm{pBabepuro-flag} \bullet \mathrm{hTERT}$, have been previously described $[9,10]$. The retroviral vectors encoding two transgenes were constructed based on these 
plasmids. pBabe-hTERT + p5 $3^{\mathrm{DD}}$ was created by subcloning the hTERT and PCR cloning the $\mathrm{p} 53^{\mathrm{DD}}$ transgenes into the EcoRI-SalI and HindIII-ClaI sites of pBabepuro, respectively, thereby replacing the puromycin selection marker. pBabe-cyclinD1+CDK4 $4^{\mathrm{R} 24 \mathrm{C}}$ was created by PCR cloning cyclin D1 and CDK4 $4^{\mathrm{R} 24 \mathrm{C}}$ into the same EcoRI-SalI and HindIII-ClaI sites. pBabe-c-Myc ${ }^{\mathrm{T} 58 \mathrm{~A}}+\mathrm{H}-\mathrm{Ras}^{\mathrm{G} 12 \mathrm{~V}}$ was created by PCR cloning the H-Ras ${ }^{\mathrm{G} 12 \mathrm{~V}}$ transgene into the HindIII-ClaI sites of pBabeblasto-c-Myc ${ }^{\mathrm{T} 58 \mathrm{~A}}$, replacing the blastocidin selection marker.

\section{Porcine transformed cell lines and tumour growth}

At one day of age, ears were notched under sterile conditions for identification purposes and the samples were used to establish a primary culture of fibroblasts. As soon as these cultures were established, they were serially infected every $48 \mathrm{hrs}$ with one of each of the retroviruses derived from the plasmids using standard approaches. RNA was isolated from the described cell lines $48 \mathrm{hrs}$ after the last infection and reverse transcribed. Resultant cDNA was used to verify the absence or presence of expression of specific transgenes by PCR amplification with one primer specific to the transgene and another specific to a transcribed region of the pBabe plasmids. Transformed cells $\left(10^{3}\right)$ per $3 \mathrm{~cm}$ plate (in triplicate) were suspended in soft agar as described and colonies $>30$ cells scored after four weeks. Transformed cells $\left(1 \times 10^{7}\right)$ were mixed with Matrigel and injected SC into the flanks of SCID/beige mice per cell line, after which tumour volumes were determined at regular intervals as described or $1 \times 10^{8}$ of cells were mixed with a similar number of isogenic control norma cells and injected orthotopically behind each ear of the donor pig treated daily with cyclosporine $(20 \mathrm{mg} / \mathrm{kg})$, azothioprine $(2 \mathrm{mg} / \mathrm{kg})$ and prednisolone $(4 \mathrm{mg} / \mathrm{kg})$.

\section{Animals and diets}

All procedures involving animals were approved by the Institutional Animal Care and Use Committee of the University of Illinois. A single Duroc gilt was used as the genomic template for producing cloned animals by nuclear transfer. Four clones, 14 months of age, were housed at the University of Illinois ISRL. Pigs were randomly assigned to one of two treatment groups: a control diet (CD) $(n=2)$ or a high-fat, high-cholesterol $(\mathrm{HFHC})$ diet $(\mathrm{n}=2)$. Control pigs were fed $2.0 \mathrm{~kg}$ of a control diet once daily in the morning and had free access to water. The second group of pigs were fed $1.5 \mathrm{~kg}$ of the HFHC atherogenic diet consisting of the control diet supplemented with $2.0 \%$ cholesterol, $17 \%$ hydrogenated coconut oil, $2.3 \%$ corn oil, and $0.7 \%$ sodium cholate once daily in the morning and had free access to water. The cholesterol diet ratios for metabolizable energy, calcium, phosphorus and the principal amino acids lysine, methionine, threonine, and tryptophan were made equivalent to the control diet.

\section{Lipid measures}

Blood samples were collected via the jugular vein from pigs fasted overnight at day 0 and every two weeks until the end of the study. Plasma was sent to Mayo Medical Laboratories for lipoprotein analysis (Rochester, MN). The lipoprotein metabolism profile reported total cholesterol and total triglycerides, and quantification of cholesterol and triglycerides in VLDL, HDL cholesterol, and LDL cholesterol.

\section{RESULTS}

\section{Porcine atherosclerosis model}

A novel porcine model of atherosclerosis was created by utilizing genomic sequence information to characterize exon 4 of porcine $A P O E$, establishing a nutritional regimen that induces the formation of atheromas, and characterizing the initial stage of atherosclerosis formation. The advantages of working with this animal model are that genetic variation is controlled for by using cloned animals, lipoprotein metabolism in swine is similar to that in humans, and the cardiovascular system, specifically coronary circulation, susceptibility to cardiovascular disease, and atherosclerotic lesion morphology is similar to humans. Unrelated pigs from ten breeds were genetically screened to identify animals that had the SNP associated with increased 
susceptibility associated with the $A P O E \& 4$ genotype. A Duroc female was selected for cloning by nuclear transfer. After reaching 14 months of age, the clones were moved to the experimental diet (Table 1). The animals received the same daily nutritional energy and were weighed to establish daily growth rates. Blood was collected weekly and was processed to establish plasma cholesterol, triglyceride and lipoprotein cholesterol levels in each animal. As shown in Table 2, after 4 weeks the total the clones fed the high fat, high cholesterol (HFHC) diet both had elevated total cholesterol, VLDL cholesterol, LDL and HDL cholesterol compared to the two control clones (CD). Thus, this diet was able to induce a rapid rise in plasma levels. In addition, these animals were shown following necropsy and histopathology to have developed atherosclerotic plaques [11].

Table 1: Diet compositions

\begin{tabular}{|lcc|}
\hline \multirow{2}{*}{ Composition of diets } & Control diet & High fat $/$ cholesterol diet \\
\cline { 2 - 3 } & Weight, g/100g & Weight, g/100g \\
\hline Corn & 85.94 & 50.89 \\
Soybean meal & 10.81 & 22.82 \\
Limestone & 1.07 & 1.4 \\
Calcium phosphate & 1.61 & 2.11 \\
Premix minerals & 0.35 & 0.45 \\
Premix vitamins & 0.2 & 0.26 \\
Tylan-40 & 0.03 & 0.03 \\
DL-methionine & 0 & 0.03 \\
Coconut oil & 0 & 17 \\
Corn oil & 0 & 2.3 \\
Cholesterol & 0 & 2 \\
Sodium cholate & 0 & 0.7 \\
\hline Energy & $3.304 \mathrm{kcal} / \mathrm{g}$ & $4.239 \mathrm{kcal} / \mathrm{g}$ \\
Daily intake & $2 \mathrm{~kg} / \mathrm{day}$ & $1.5 \mathrm{~kg} / \mathrm{day}$ \\
\hline
\end{tabular}

Table 2: Plasma cholesterol and triglyceride levels and lipoprotein cholesterol levels in control and high fat, high cholesterol fed swine.

\begin{tabular}{|lcccc|}
\hline \multicolumn{1}{|c}{ Serum value (mg/dL) } & \multicolumn{2}{c}{ Week 0 } & CD2 \\
Total cholesterol & HFHC1 & HFHC2 & CD1 & 82 \\
VLDL cholesterol & 73 & 84 & 79 & 3 \\
LDL cholesterol & 3 & 1 & 2 & 46 \\
HDL cholesterol & 40 & 53 & 43 & 33 \\
Total triglyceride & 30 & 30 & 34 & 43 \\
VLDL triglyceride & 33 & 35 & 41 & 34 \\
LDL triglyceride & 27 & 20 & 33 & 5 \\
& 2 & 4 & 4 & CD2 \\
& & & Week 4 & \\
Total cholesterol & HFC1 & HFHC2 & CD1 & 63 \\
VLDL cholesterol & 439 & 381 & 61 & 7 \\
LDL cholesterol & 32 & 29 & 6 & 30 \\
HDL cholesterol & 337 & 300 & 30 & 26 \\
Total triglyceride & 70 & 52 & 25 & 26 \\
VLDL triglyceride & 21 & 30 & 24 & 19 \\
LDL triglyceride & 9 & 19 & 17 & 4 \\
\hline
\end{tabular}




\section{Generation of porcine cells harbouring genetic changes commonly found in human cancers}

To establish a genetically malleable porcine model of cancer, normal porcine cells were genetically engineered in culture to recapitulate changes commonly found in human cancers, and when returned back to the host (isogenic) swine formed tumours [12]. Primary skin cultures, confirmed to be vimentin-positive via western blot and displayed a fibroblast morphology were established from ear notch biopsies. Fibroblasts were chosen primarily because of the ease with which they can be isolated, the existence of established cultured conditions for these cells, and the fact that human fibroblasts can be converted to a tumourigenic state by disrupting the aforementioned p53, Rb, c-Myc, Ras and telomerase pathways. These early passage porcine fibroblasts were sequentially and rapidly infected over the course of six days with three retroviruses co-expressing pairs of available human and murine versions of the cDNAs for $h T E R T$ and $p 53^{D D}$, cyclin D1 and $C D K 4^{R 24 C}$, and lastly, $c-M y c^{T 58 A}$ and $H-R_{a S}{ }^{G 12 V}$. RNA was isolated from the resulting polyclonal population, termed $6510 \mathrm{~T}$, and subjected to RT-PCR with one primer anchored in the transgene and another in an accompanying vector-specific untranslated region to specifically amplify the transgenic mRNAs. Using this approach, all six transgenes were verified to be expressed in the pig fibroblast cells (Table 3).

Table 3: Genetic conversion of normal porcine fibroblasts to a tumourigenic state by disrupting pathways commonly altered in human cancers.

\begin{tabular}{|c|c|c|c|}
\hline \multirow{2}{*}{$\begin{array}{l}\text { Genetic element } \\
\text { ( } \Delta \text {, deletion of single } \\
\text { gene from } \\
\text { transfection) }\end{array}$} & \multicolumn{3}{|c|}{ Porcine fibroblast phenotype } \\
\hline & $\begin{array}{c}\Delta \text { Transgene } \\
\text { expression } \\
\text { (RT-PCR) }\end{array}$ & Colony number & $\begin{array}{l}\text { In vivo tumour } \\
\qquad\left(1^{6}\right)\end{array}$ \\
\hline Vector & No & 0 & No \\
\hline$\Delta \mathrm{p} 53^{\mathrm{DD}}$ & No & 900 & Yes \\
\hline$\Delta$ Cyclin D1 & No & 0 & NO \\
\hline$\Delta \mathrm{CDK} 4^{\mathrm{R} 24 \mathrm{C}}$ & No & 0 & Yes (small) \\
\hline$\Delta \mathrm{Myc}^{\mathrm{T} 58 \mathrm{~A}}$ & No & 400 & No \\
\hline$\Delta \operatorname{Ras}^{\mathrm{G} 12 \mathrm{~V}}$ & No & 0 & No \\
\hline$\triangle \mathrm{hTERT}$ & No & 1,000 & Yes \\
\hline $\begin{array}{l}\text { p53 } 3^{\mathrm{DD}}, \text { Cyclin D1, } \\
\text { CDK4 } 4^{\mathrm{R} 24 \mathrm{C}}, \mathrm{Myc}^{\mathrm{T} 58 \mathrm{~A}}, \\
\text { Ras }^{\mathrm{G} 12 \mathrm{~V}}, \text { hTERT }\end{array}$ & Yes, all expressed & 4,000 & Yes \\
\hline
\end{tabular}

Confirmation of appropriate transgene expression by RT-PCR of primary porcine fibroblast cultures. Established cultures were infected with retroviruses encoding hTERT, p53DD, cyclin D1, CDK4R24C, c-MycT58A, and $\mathrm{H}$-RasG12V, or combinations of five of these six cDNAs (cell lines $\triangle \mathrm{hTERT}, \Delta \mathrm{p} 53 \mathrm{DD}, \Delta$ cyclin D1, $\Delta \mathrm{CDK} 4 \mathrm{R} 24 \mathrm{C}$, $\Delta$ MycT58A and $\Delta$ RasG12V, which lack the indicated transgene), or as a negative control, empty vectors (vector).

Engineered genetic changes endowed porcine cells with the ability to grow in an anchorage-independent fashion in soft agar, one of the most rigorous in vitro assays for the transformed growth characteristic of cultured cancer cells. Transformed porcine fibroblasts, or as a negative control, cells serially infected with vectors 
lacking these transgenes, were seeded in soft agar and three weeks later colony growth was assessed. Only cells expressing all six transgenes were capable of robust transformed cell growth (Table 3). To address the requirements of transformation of porcine cells, all combinations of five of the six transgenes were introduced into primary normal cells by sequential infection with two retroviruses encoding four different transgenes, followed by infection with a retrovirus encoding only one transgene. The resultant six polyclonal populations (denoted by indicating the missing transgene) were confirmed by RT-PCR to express the desired combinations of five transgenes. When these populations were assayed for growth in soft agar, loss of any one transgene was found to reduce the number of colonies. As with cells from the first animal, expression of all six transgenes was again found to be required for robust transformed cell growth in soft agar. To determine whether expression of the described six transgenes was sufficient to drive normal porcine fibroblasts to a tumourigenic state, the transformed cells were injected SC into immunocompromised mice, and tumour growth was monitored over time. These cells rapidly formed tumours at the site of injection, reaching maximum tumour burden within ten days in all animals, whereas vector control cells failed to grow in this fashion. These cells maintained transgene expression in vivo, as assessed by RT-PCR, and formed vascularized tumours characterized by poorly differentiated cells with large nuclei and multiple nucleoli. To address the requirements for tumourigenic growth, porcine cells were injected into immunocompromised mice. These cells did not form tumours in the absence of cyclin D1, c-Myc ${ }^{\mathrm{T} 8 \mathrm{~A}}$, or HRas $^{\mathrm{Gi}}{ }^{\mathrm{V}}$, but could still form tumours when either $\mathrm{p} 53^{\mathrm{DD}}$, hTERT, or CDK4 proteins was omitted. Thus, multiple transgenes are also needed for tumour growth, although interestingly, not as many as required for robust transformed growth in vitro. Porcine fibroblasts isolated from ten unrelated outbred pigs infected with retroviruses encoding the same six transgenes were shown to all form tumours in mice, with an average latency period of three days [12]. Whether expression of these six proteins would drive other types of porcine cells (embryonic kidney, porcine testes and kidney epithelial cells) to a tumourigenic state was also tested. The six transgenes were able to similarly induce tumours when injected into mice. In conclusion, inactivation of the tumour suppressors $p 53$ and $R b$ concomitant with the activation of $c-M y c, R a s$, and telomerase is reproducibly tumourigenic in multiple porcine cell types, even when the cells are isolated from an outbred population [12].

\section{Ataxia-telangiectasia (A-T)}

This genetic disorder causes cerebellar degeneration, immune deficiency, cancer predisposition, chromosomal instability and radiation sensitivity. Among the mutations responsible for $\mathrm{A}-\mathrm{T}, 85 \%$ represent truncating mutations that result in the production of shorter, highly unstable forms of ATM (AT-mutated) protein leading to a null ATM phenotype. Several ATM-deficient mice have been created however none reflect the extent of neurological degeneration observed in humans. In an attempt to identify an alternative animal model, we have characterized the porcine ortholog of $A T M(p A T M)$ [13]. When compared to the human ATM (hATM), the $p A T M$ showed a high level of homology in the coding region, particularly in the regions coding for functional domains, and had extensive alternative splicing of the $5^{\prime} \mathrm{UTR}$, characteristic for the human ATM mRNA. Overall, the porcine ATM protein consists of 3,058 aa and is highly homologous to the hATM protein (88\% identity and $93 \%$ positive). Among all the non-primate mammalian species, the human protein has the highest homology with pATM, with identity greater than $92 \%$ in the FAT and PIK functional domains. 
Six different 5' UTRs resulting from alternative splicing of the first three exons were identified. The porcine 5' UTRs varied in size, had multiple ATG codons and different secondary structures, supporting the possibility of complex transcriptional regulation. In contrast with $h A T M$, alternative splicing of the $5^{\prime}$ ' region involved not only the 5' UTR but also the coding region. Three of the six transcripts demonstrated splicing of the first coding exon, altering the translation start, and giving rise to a putative protein lacking the N-terminus substrate binding domain (82-89 aa) involved in activation of human 553 and BRCA1 pathways. Real-time PCR analysis revealed variable expression levels of total $A T M$ transcripts in individual tissues (Fig. 1). Although each splice variant was ubiquitously expressed among the tissues, differences in the relative abundances of specific 5' UTRs were observed. The longest splice variant represented no less than $60 \%$ of the total $A T M$ transcript in each tissue examined.

\section{Discussion}

In the future, we will continue to investigate the effects of complex gene and environmental interactions on complex phenotypes. This paper shows how genomics can be used to develop animal models of human disease based on a single nucleotide polymorphism (atherosclerosis), disrupting regulatory pathways (tumourigenesis) and differences in splice variants (AT). With regard to atherosclerosis, additional studies will be performed with longer feeding trials to further characterize the developmental process of atherosclerosis and establish a timeline of progression. Once this is accomplished, intervention procedures can be performed and the most appropriate treatment for a particular stage of atherosclerosis can be determined. In addition, as our understanding of the genetics of atherosclerosis increases, genetic diagnosis will become increasingly important. One of the greatest potential benefits of genetic studies is testing for susceptibility. Once the genes contributing to common forms of the disease have been identified, along with the particular mutations involved, DNA-based tests will contribute to our ability to assess risk.

We also demonstrated that porcine cells can be genetically engineered to perturb pathways commonly altered in human cancers and become tumourigenic and when returned back to the host animal, provide the first method of genetically inducing tumours in a large mammal. The pig has a number of clear advantages that make it ideal for preclinical imaging studies, as well as studies on hyperthermia, radiation, or photodynamic therapy of tumours. First, the changes required to make porcine cells tumourigenic are more similar to those needed in human compared to murine cells, and hence tumourigenesis in pigs may be more similar to the process in humans. Second, like companion animal models of cancer, the resultant tumours in pigs can be grown to large sizes, ideal for a number of preclinical applications. Third, in this study the porcine tumours were genetically engineered, and hence it is possible to tailor-make tumours of a defined background, unlike with companion animal models. Fourth, as different cell types injected at different anatomical sites all yielded tumours, it should be possible to generate many different types of tumours essentially anywhere in the body. Fifth, the entire procedure is quite simple and nearly as rapid as existing mouse xenograft models. The ability to genetically generate experimentally malleable tumours of a size and genetic makeup akin to those found in humans rapidly, and at will in the pig, can now be exploited with relative ease as a relevant model for preclinical studies of human cancers.

Finally, an extremely important role of alternative splicing is expanding protein diversity. Approximately $75 \%$ of human genes are alternatively spliced [14] and the 
species-specific alternative splicing observed in human and pig ATM represents the most common type of splicing in mammalian species. The alternative splicing events that affect the coding sequence change protein functions and thus provide proteomic diversity. The multiple splice variants of porcine $A T M$ gene that were identified in this study could possibly be translated into various proteins with yet unknown functions. Overall, the extensive alternative splicing of the porcine ATM gene resembles complex AS in humans and could impact differences observed between mice and humans with regards to the onset of $\mathrm{A}-\mathrm{T}$.

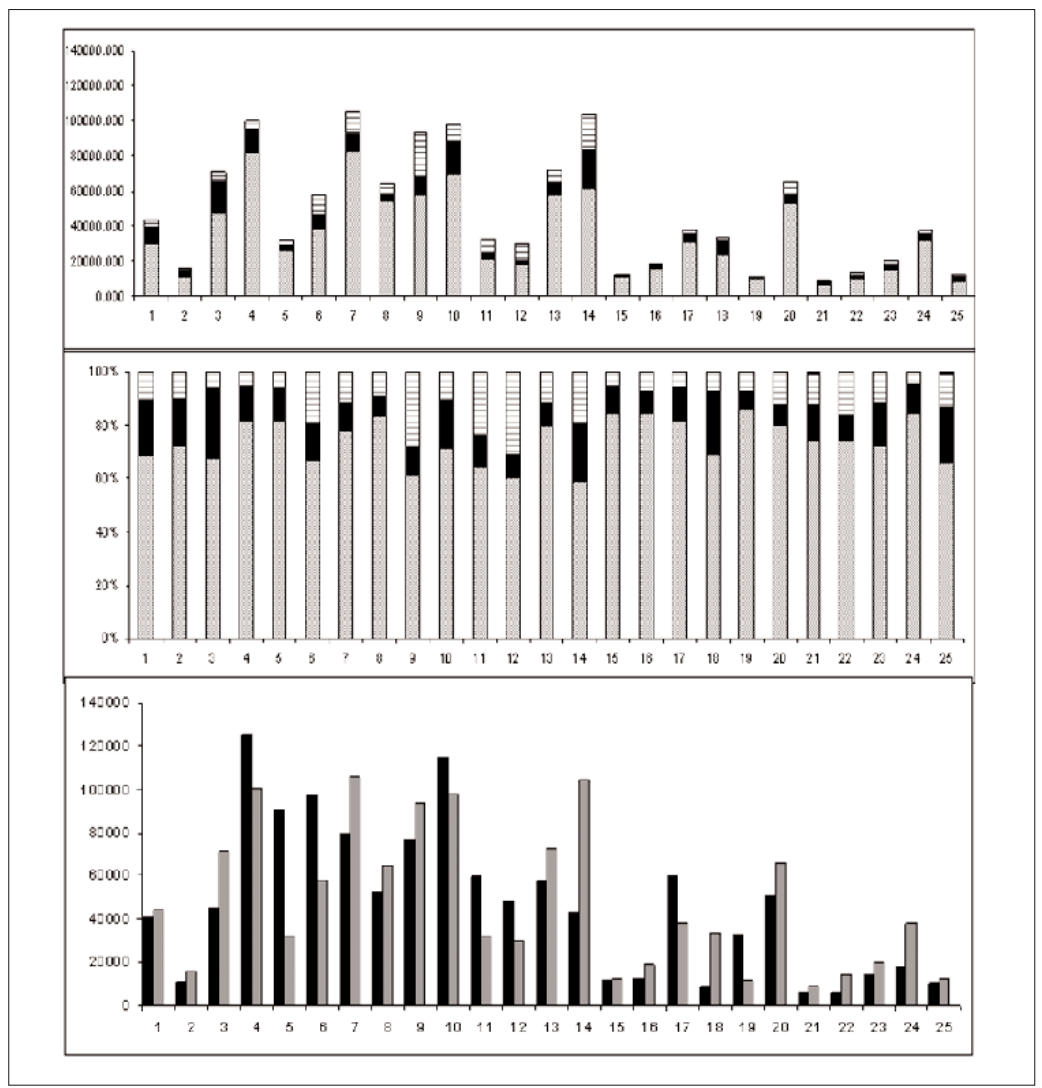

Fig. 1:Tissue expression of porcine ATM splice variants (SV1, SV2 and SV3).

RT-PCR was used to quantify cDNA from different porcine tissues for SV1, SV2, and SV3 transcripts. The TaqMan assay for quantitative RT-PCR was used to determine expression levels. Expression was normalized by using the level of $18 \mathrm{~s}$ rRNA. Grey bars are for SV1 transcripts, black - for SV2 transcripts, and bars with stripes are for SV3 transcripts. Analysed tissues are: 1, skin; 2, fibroblasts; 3, spinal cord; 4, brain; 5, adrenal gland; 6, mandibular lymph node; 7, mesenteric lymph node; 8 , spleen; 9 , ovary; 10 , uterus; 11 , testis; 12 , fat; 13 , bone morrow; 14 , heart; 15 , muscle; 16 , kidney; 17 , thymus; 18 , thyroid; 19, lung; 20, trachea; 21, small intestine; 22, colon; 23, stomach; 24, liver; and 25, bladder. Upper Panel: expression profile is given as copy numbers for each SV per $25 \mathrm{ng}$ of amplified cDNA. Middle Panel: percentage (\%) of each SV from the total amount of SV1, SV2 and SV3 ATM transcripts. Lower Panel: total amount of ATM transcripts. Black bars represent the copy number of transcripts obtained by TaqMan assay using the exon6-exon7 junction as a probe. Dotted bars represent the copy number calculated as the sum of transcripts detected with SV1, SV2 and SV3 TaqMan assays in the same tissue. 


\section{Conclusions}

Comparative genomics can provide insights into the development of "ideal" animal models for addressing human diseases. In addition, tools (targeted gene manipulation, and NT cloning) are emerging to create comparative phenotypic models, however, more phenotypic metrics are required to recapitulate the human condition. The pig genome $\mathrm{x}$ environment $(\mathrm{G} \times \mathrm{E}$ ) nutritional model of human cardiovascular disease has been validated and supports intervention strategies. Porcine drug metabolism is more relevant to human FDA studies than murine [8]. Targeting porcine pathways associated with human cancer provides a genetically malleable tumour model for pre-clinical applications. Both sequence diversity and splice variants contribute to the complex neurological disease phenotypes. Recombineering supports the development of specific allelic and SV lines for the study of developmental genes.

\section{ACKNOWLEDGEMENTS}

This work was supported in part by USDA/NRI-CSREES grantAG2001-35205-11698, USDA-ARSCooperative Agreement AG58-5438-2-313, DOD Training Grant W81XWH-06-1-0437 and the AT Children's Project.

\section{REFERENCES}

1 National Vital Statistics Reports. 2002. Estimated life expectancy at birth in years, by race and sex: Deathregistration States, 1900-28, and United States, 1929-2000.

2 National Vital Statistics Reports. 2000. Deaths: Leading Causes for 2000.

3 Swanson KS, Mazur MJ, Vashisht K, Rund LA, Beever JE, Counter CM, Schook LB: Genomics and clinical medicine: Rationale for creating and effectively evaluating animal models. Exp Biol Med 2004;229:866-875.

4 Lusis AJ, Fogelman AM, Fonarow GC: Genetic basis of atherosclerosis: Part i: New genes and pathways. Circulation 2004;110:1868-1873

5 de Knijff P, van den Maagdenberg AM, Frants RR, Havekes LM: Genetic heterogeneity of apolipoprotein e and its influence on plasma lipid and lipoprotein levels. Hum Mutat 1994;4: 178-194.

6 Dewhirst MD, Thrall D, Macewen E: Spontaneous Pet Animal Cancers, In Teicher BA (ed.): Tumor Models in Cancer Research, Totowa, NJ, Humana Press, 2000, pp 565-589.

7 Tumbleson M, Schook LB (ed.): Advances in Swine in Biomedical Research. New York, NY, Kluwer Academic, 1996.

8 Pollock CB, Rogatcheva MB, Schook LB: Comparative genomics of xenobiotics metabolism: a porcinehuman PXR gene comparison. Mamm Genome 2007;18:210-219.

9 Hahn WC, Dessain SK, Brooks MW, King JE, Elenbaas B, Sabatini DM, DeCaprio JA, Weinberg RA Enumeration of the simian virus 40 early region elements necessary for human cell transformation. Mol Cell Biol 2002;22:2111-2123.

10 Yeh E, Cunningham M, Arnold H, Chasse D, Monteith T, Ivaldi G, Hahn WC, Stukenberg PT, Shenolikar S, Uchida T, Counter CM, Nevins JR, Means AR, Sears R: A signalling pathway controlling c-Myc degradation that impacts oncogenic transformation of human cells. Nat Cell Biol 2004;6:308-318.

11 Mazur M, Pettigrew J, Zachary J, Schook LB: Dietary cholesterol induced atherosclerosis in genetically pre-disposed cloned pigs. Under review. 
12 Adam SJ, Rund LA, Kuzmuk KN, Zachary JF, Schook LB, Counter CM: Genetic induction of tumorigenesis in swine. Oncogene 2007;26:1038-1045

13 Rogatcheva MB, Fritz KL, Rund LA, Pollock CB, Beever JE, Counter CM, Schook LB: Characterization of the porcine ATM gene: Towards the generation of a novel 3 non-murine animal model for AtaxiaTelangiectasia. Gene doi:10.1016/j.gene.2007.08.014, in press.

14 Johnson JM, Castle J, Garrett-Engele P, Kan Z, Loerch PM, Armour CD, Santos R, Schadt EE, Stoughton, $\mathrm{R}$, Shoemaker, DD: Genome-wide survey of human alternative pre-mRNA splicing with exon junction microarrays. Science 2003;302:2141-2144.

Dr. Lawrence B. Schook, PhD, Institute for Genomic Biology, University of Illinois, Room 2109, 1206 W. Gregory Drive, Urbana, IL 61801, USA.

E-mail: schook@uiuc.edu 